\title{
Review Article \\ Optical Properties of the Self-Assembling Polymeric Colloidal Systems
}

\author{
Alexandra Mocanu, Edina Rusen, and Aurel Diacon \\ Department of Polymer Science, University Politehnica of Bucharest, 149 Calea Victoriei, 010072 Bucharest, Romania \\ Correspondence should be addressed to Edina Rusen; edina_rusen@yahoo.com
}

Received 11 March 2013; Revised 17 June 2013; Accepted 20 June 2013

Academic Editor: Haojun Liang

Copyright ( 2013 Alexandra Mocanu et al. This is an open access article distributed under the Creative Commons Attribution License, which permits unrestricted use, distribution, and reproduction in any medium, provided the original work is properly cited.

In the last decade, optical materials have gained much interest due to the high number of possible applications involving path or intensity control and filtering of light. The continuous emerging technology in the field of electrooptical devices or medical applications allowed the development of new innovative cost effective processes to obtain optical materials suited for future applications such as hybrid/polymeric solar cells, lasers, polymeric optical fibers, and chemo- and biosensing devices. Considering the above, the aim of this review is to present recent studies in the field of photonic crystals involving the use of polymeric materials.

\section{Photonic Crystals}

Nanomaterials with well-defined 3D repetitive arrays have found application in photonic devices, sensors, selective membranes, and even microchips.

Compared to a classic crystalline array, colloidal spherical particles replace the molecules, atoms, or ions in hexagonal close packed (HCP) structure or cubic close packed (CCP) structures [1-3] (Figure 1).

Colloidal particles are able, through self-assembly processes, to be arranged into $3 \mathrm{D}$ structures leading to colloidal crystals. The resulting materials revealed interesting properties, presenting similar optical characteristics with synthetic opal and photonic crystals (Figure 2). One of the most facile routes for obtaining photonic crystals is based on the employment of polymer colloids with self-assembling properties [3-5].

A photonic crystal is a periodic nanostructured material that influences the propagation of electromagnetic waves in a similar manner as a semiconductor does for electrons [3].

Although photonic crystals have been studied since 1887, with one of the pioneers being Rayleigh [6], the term has been introduced only in 1987, after the papers of two physicist working independently Yablonovitch and John $[7,8]$.
In order to understand the behavior of photons inside a photonic crystal it is possible to compare it with the motion of electrons and vacancies in a semiconductor material as mentioned above. A proper example of photonic crystals occurring in nature is opals, which contain a natural periodic microstructure of silica microspheres of $15-900 \mathrm{~nm}$, responsible for their iridescent color [9]. This structure allows the propagation of the photons through the crystal, while the interaction between the silica spheres and light makes possible the formation of allowed and forbidden energy bands, also known as band gaps or stop bands [8]. Thus, the propagation of the electromagnetic waves is forbidden for specific wavelengths.

Therefore, a photonic crystal is a powerful tool for light modulation in certain directions of space. Exploiting these properties, photonic crystals have been used in many optical applications, such as the use in sensors and monitors [10, $11]$, bioassays $[12,13]$, colour displays $[14,15]$, solar energy $[16]$, and lasers [17, 18]. For all applications involving PCs one of the key parameters that have to be controlled is the band-gap position. The stop-band value depends primarily on three factors [19-22]: the refractive index contrast between the dielectric components (solid and air usually), the lattice constant (the spacing between the spheres), and the filling 


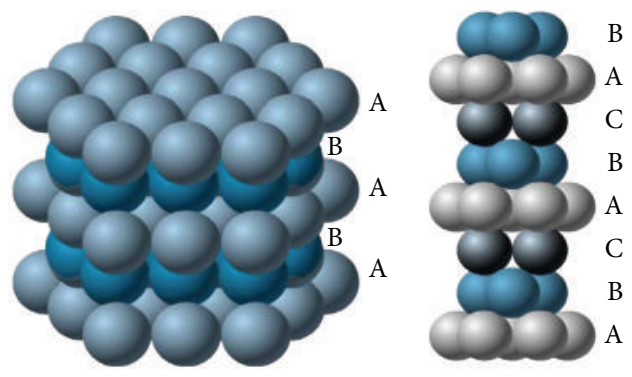

FIgURE 1: Three-dimensional arrays: (a) HCP, (b) CCP [3].

factor (volume of the colloids related to the volume of surrounding media). In addition, one of the most challenging applications consists in fabricating photonic crystals with $3 \mathrm{D}$ band gap around $1.55 \mu \mathrm{m}$ representing the wavelength used in optical fibers [23].

\section{Polymeric Photonic Crystals}

In order to obtain colloidal crystals, the most common methods consist in surfactant-free emulsion polymerization $[5,24]$, precipitation polymerization $[25,26]$, dispersion polymerization [27], and seeded emulsion polymerization [28]. The absence of surfactants leads to polymer latex of high purity, and at the same time, the polymerization parameters (temperature, initiator and monomer concentrations, stirring, etc.) are facile to control. The final monodisperse polymer particles represent a class of materials that can be easily functionalized on the surface, therefore, increasing the number of possible applications [1].

As it was specified in the previous section, colloidal crystals represent HCP or CCP structures, consisting of colloidal microspheres ranging from $100 \mathrm{~nm}$ to $1 \mu \mathrm{m}$. Depending on the arrangement parameters the photons are forbidden to propagate inside colloidal crystals in certain directions, for specific wavelengths. If the lattice potential is strong enough, the gap might extend to all possible directions, resulting in a complete band gap [29]. This property allows the control of the light that interacts with the colloidal crystal.

Taking this into account, it is easy to realize that one of the most important optical properties of the colloidal crystals consists in light diffraction according to Bragg's law.

2.1. Bragg's Law. In order to better understand the phenomenon that occurs inside a polymer photonic crystal, it is necessary to analyze the path of an incident beam that travels through the crystal planes.

The diagram below [30] (Figure 3) explains the pathway the beam B, which represents the extra distance traveled by beam A. Perpendicular lines, (l) lines, were drawn to both rays. Following this method two right triangles were obtained [30].

The angle, marked $\theta$, also called Braggs angle can be calculated from these triangles as $\sin \theta=s / d$. Therefore, the extra distance is $2 s$ long or $2 d \sin \theta$ long. This value is equal with a number of wavelengths $(n)$ for a strong beam (Bragg's law)

$$
n \lambda=2 d \sin 0 .
$$

When the Bragg equation is satisfied, the angle formed by the diffracted beam with the crystal plane is equal to the one between by the incident beam and the same crystal plane. In this situation, diffraction is similar to a reflection from a mirror (Braggs mirror effect), where the angle of incidence is identical to the angle of reflection [30].

In the case of molecular, atomic, or ionic crystals, the interplanar spacing, $d$, is in the range of $\AA$, thus having the capacity of diffracting $X$-rays. In the case of colloidal crystals, $d$ is in the range of $100 \mathrm{~nm}$, which implies the diffraction of light with wavelengths of the same order of magnitude (400-800 nm). Therefore, the colloidal crystal will diffract light with wavelengths corresponding to violet-blue-green and transmit it in the complementary radiation (orange-red) of visible light.

2.2. Bragg's Law on Colloidal Crystal. The examination of a colloidal dispersion (Figure 4) during and after solvent evaporation allows the observation of iridescence in different areas of the visible spectrum due to diffraction of the light in accordance with Bragg's law [31].

The optical microscopy shows that the distance between the defaults due to water evaporation is only $150-200 \mu \mathrm{m}$, which proves the high quality of the film, respectively, of the obtained opal [32]. The crystallization process reveals permanent changes in the color of the colloidal dispersion. In the first step, the dispersion is white due to Mie scattering, and $d$ is larger than polymer particles [33]. In the second step, the colloidal dispersion changes its colors as a consequence of reducing the interplanar distance.

Figure 5(a) shows a well-ordered close packed structure with a triangular arrangement that can be attributed to planes (111) of a CCP system [9, 31, 34]. Moreover the (fast fourier transform) FFT of the image (Figure 5(b)) displays sharp peaks confirming the existence of long-range crystalline order.

According to Bragg's law, the change of the angle between the incident radiation and photonic crystal leads to notable changes of the reflected wavelength, respectively, of the observed color (Figure 6) [35, 36].

UV-VIS spectra of polymer photonic crystals indicate in many cases sharp peaks, which confirm the existence of highly ordered structures, due to Braggs reflection of periodic arrangement of the polymer particles [37-40].

Figure 7(b) represents the reflection spectra of polymer colloidal crystals with different size particles $(178 \mathrm{~nm}$, $211 \mathrm{~nm}, 235 \mathrm{~nm}$, and $270 \mathrm{~nm}$ ) corresponding to photograph (Figure 7(a)) [41].

Moreover, these materials show structural color changes (Figure 8) under mechanical stress. In this case, the photonic crystal film, deposited on a plastic plate, changed from red to green as result of the modification of the interplanar distance, which is thus a change in the wavelength of the diffracted light [37]. 


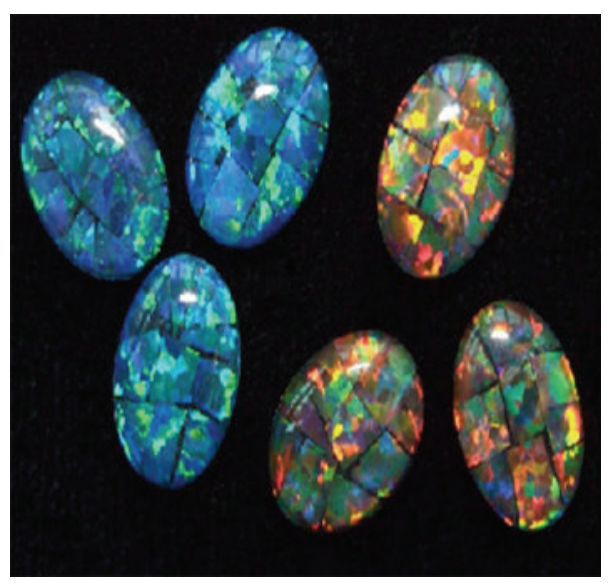

(a)

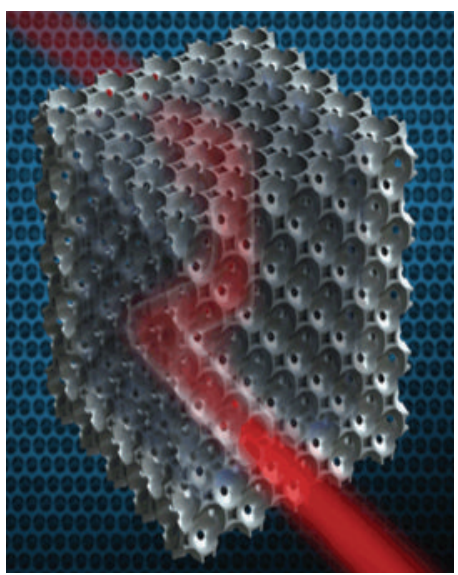

(b)

FIgURE 2: (a) Synthetic opal; (b) structure of a photonic crystal [4].

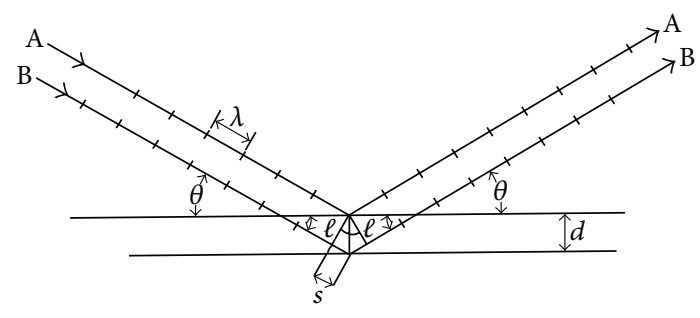

FIGURE 3: The diffraction of the light by crystal planes [30].
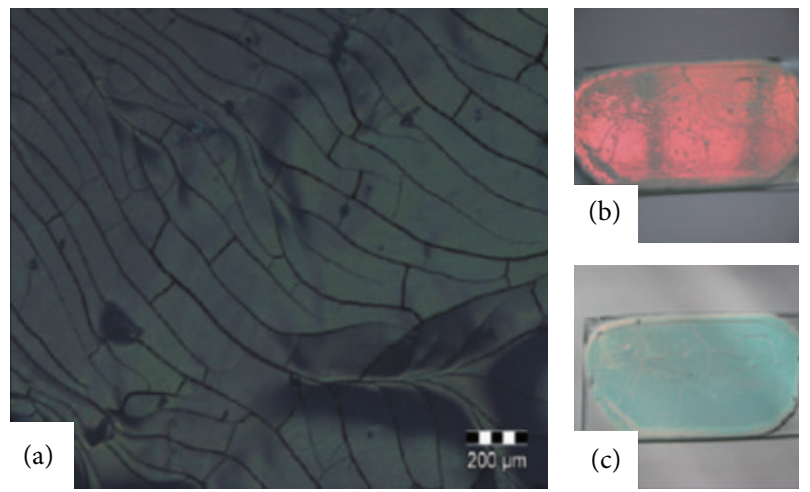

FIGURE 4: Light diffraction: iridescence of polymer colloidal spheres: (a) optical microscopy; (b) photo recorded at an angle of $45^{\circ}$ following thermal treatment; (c) photo recorded at an angle of $90^{\circ}$ following thermal treatment [31].

Although, until now, polymer photonic crystals were not expected to exhibit full stop bands, they were easy to prepare, leading to materials that allow the control of the light that interacts with the crystalline colloidal arrays $[3,42]$.

\section{Applications of the Polymeric Photonic Crystals}

In the last years, polymeric photonic crystals found application in optical devices [43-45] and sensors [46] or as

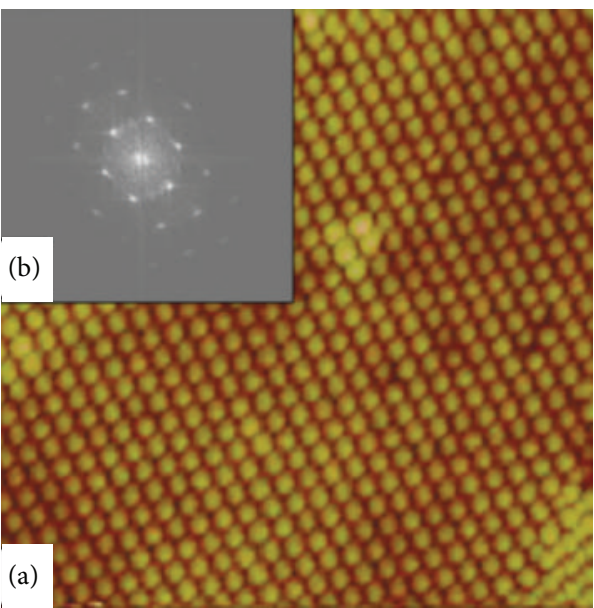

FIGURE 5: (a) AFM image of the top surface of the opal and (b) FFT of the image [31].

templates for other interesting porous-media applications taking advantage of their optical properties, composition, or morphology [26, 47, 48].

3.1. Templates for Porous-Media Applications. Polymer colloidal crystals without complete band gaps can still be used as templates to create new structures with complete band gaps [49].

The first step in obtaining inverse opal is to arrange by self-assembly polymer colloidal spheres into periodic arrays (Figure 9). The voids between the polymer particles can then be filled with different solutions that play the role of matrix. After this procedure, the polymer spheres are removed by dissolution or calcination leaving air spaces in the matrix. The resulting system is a photonic crystal with different optical properties (incremental shift and broadening with respect to those in spectra of parent opals) (Figure 10).

Waterhouse et al. [9] obtained inverse opal structures by depositing inorganic particles such as silica, titania, and ceria respectively, by a sol-gel method in the voids of 


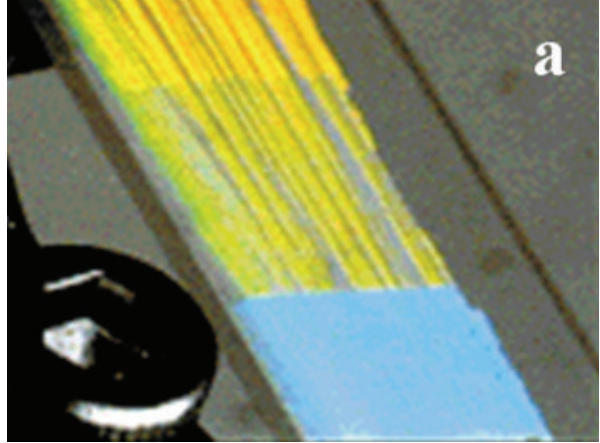

(a)

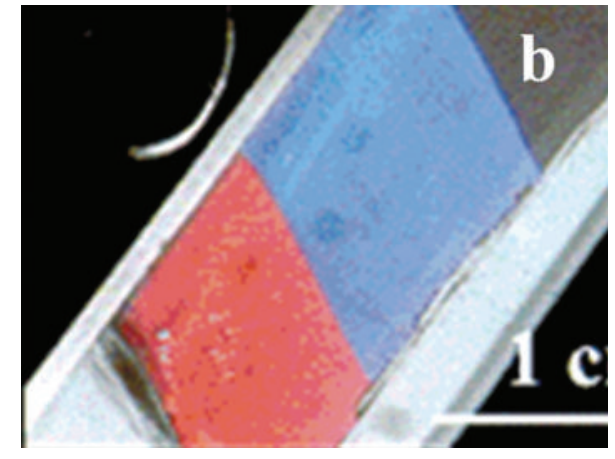

(b)

FIGURE 6: Reflected colors of colloidal dispersions registered at different angles [36].

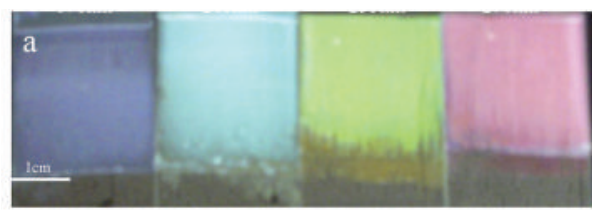

(a)

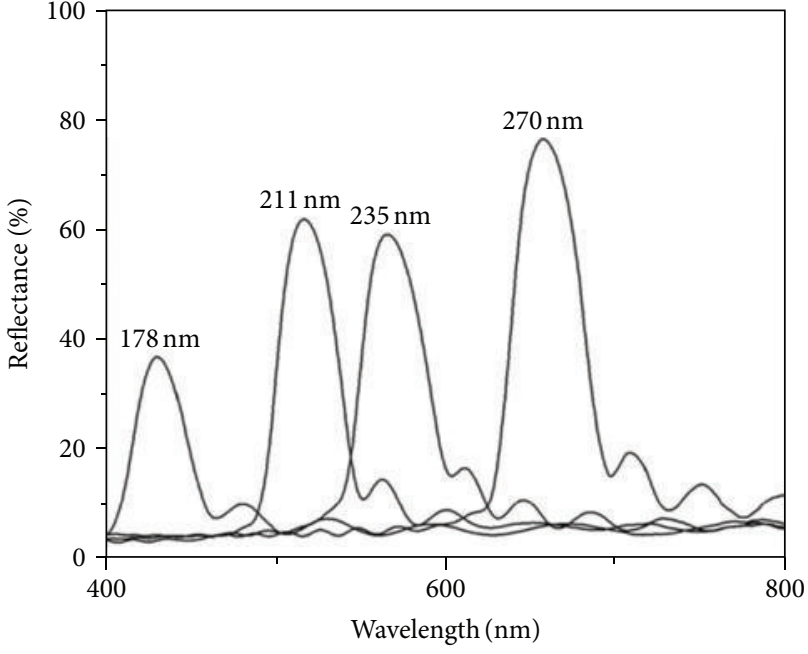

(b)

FIgURE 7: Polymer colloidal dispersions: (a) photograph taken at normal incidence; (b) reflection spectra [41].

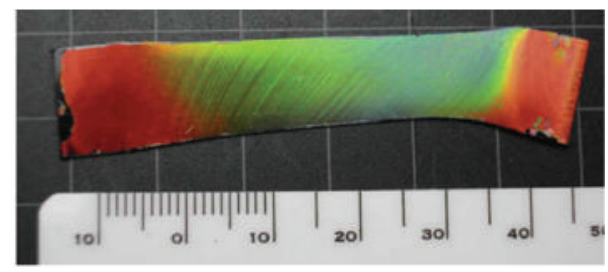

FIGURE 8: Structural color change in a crystal film after mechanical stress [37].

a polymeric photonic film. The poly(methyl methacrylate) polymer self-assembled spheres (Figure 11(b)) were removed by calcination at $550^{\circ} \mathrm{C}$. The process resulted in obtaining a macroporous matrix with CCP ordered air spheres separated by inorganic nanoparticles according to the layout presented below (Figure 11(a)). The macroporous materials formed were characterized by SEM analysis in comparison with the polymer poly(methyl methacrylate) templates in order to put into evidence the inverse opal structures highlighted in Figure 11(c) [9].

Macroporous 3D structures were obtained also by a dipcoating procedure explained by Moon et al. [50]. In this study,

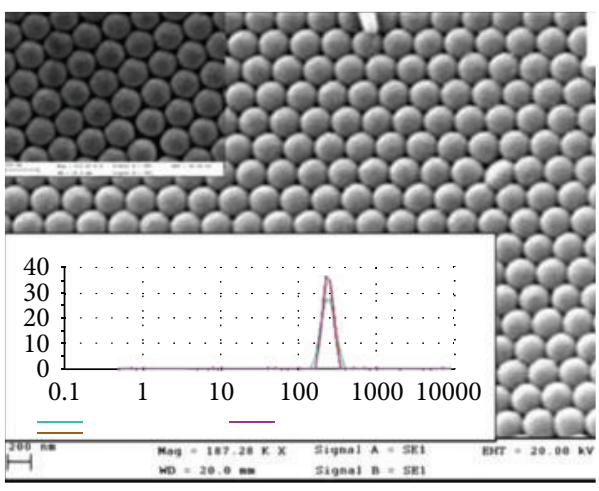

FIGURE 9: Polymer colloidal crystal obtained by soap free emulsion polymerization and DLS spectrum [65].

they obtained porous cylinders with interconnected air voids using commercial cylindrical glass fiber of 60 and $100 \mu \mathrm{m}$, respectively.

The fiber was dipped into the colloidal dispersion. The colloidal poly(styrene) particles self-assembled in a CCP 


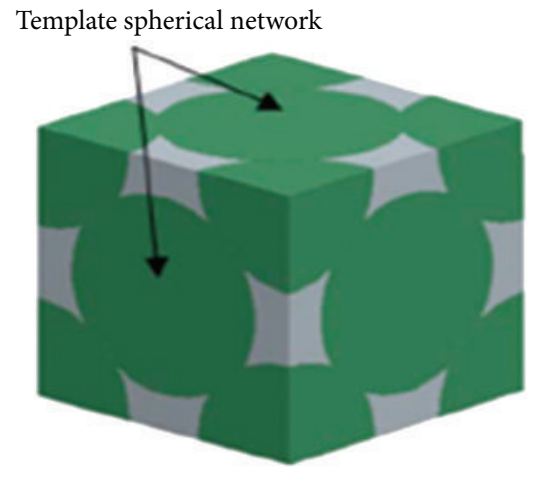

(a)

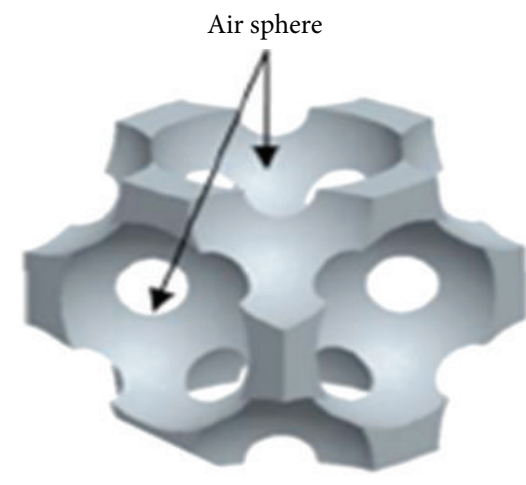

(b)

Figure 10: Unit cell of an infiltrated opal (a); unit cell of an inverse opal (b) [66].

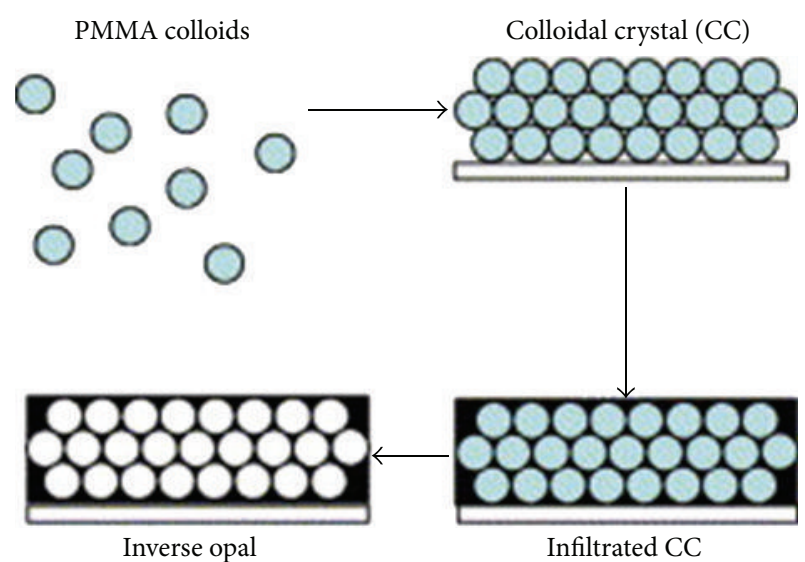

(a)

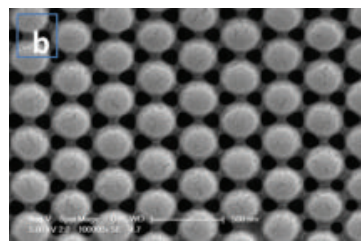

(b)

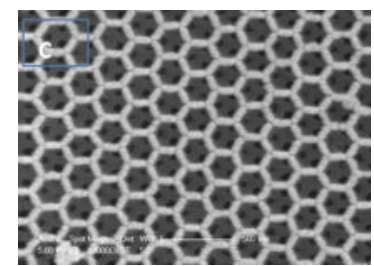

(c)

FIgURE 11: Fabrication scheme of an inverse opal (a); poly(methyl methacrylate) colloidal spheres (b); $\mathrm{TiO}_{2}$ inverse opal (c) [9].

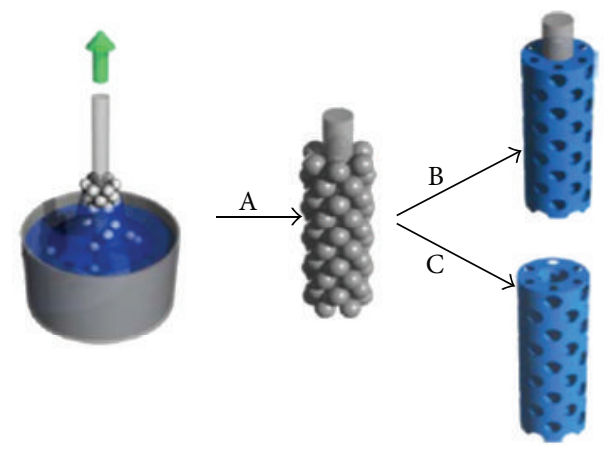

FIgURE 12: Schematic illustration of the self-assembly of colloidal spheres on a cylindrical glass fiber (A) and the inverted structure with glass fiber core (B), respectively, with hollow core (C) [50].

structure on the cylindrical glass substrate during the evaporation of the solvent. Then, a solution of polyurethane was infiltrated between the poly(styrene) arranged spheres and polymerized. The macroporous cylindrical structure was obtained on a glass fiber support by dissolving the poly(styrene) spheres. By removing the inner core an inverted porous structure with hollow core was obtained (Figures 12 and 13).
Moreover, the authors demonstrated that these structures have a controllable pore size by using colloidal particles with different dimensions. Therefore, these porous fibers are suitable for separation of membranes or catalyst support [50].

Kalinina et al. [51] proposed a new method in order to obtain materials with optically sensitive matrix starting from monodisperse core-shell colloidal particles. In the first step of this study, the particles that possess a hard core of poly(methyl methacrylate) and a relatively soft shell of poly(butyl methacrylate) are self-assembled in a crystalline array. The film is annealed at a temperature that is above the glass transition temperature $T_{g}$ of the polymeric shell and below the $T_{g}$ of the polymeric core. At this temperature, the shell fills the air spaces between the colloidal particles, leaving a matrix with intact cores and totally different optical properties towards the initial polymer film. The incorporation of a fluorescent dye during the polymerization process of the shell forming polymer resulted in a substantial reduction in optical contrast between the particles and the matrix [51].

The type of the matrix often influences the specific applications of inverse opal structures. A polymer matrix formed by poly(methyl methacrylate), polystyrene, polyurethane, or electroconducting polymers is suitable for optical devices. For example, polypyrrole, polythiophene, or polyaniline films 


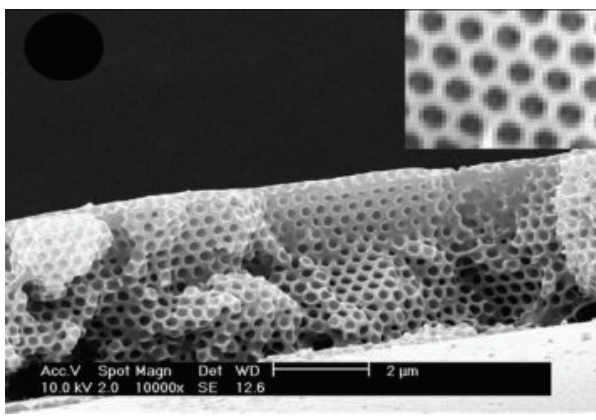

(a)

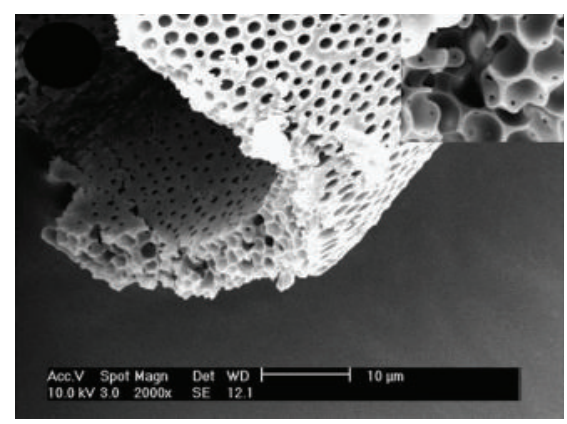

(b)

FIGURE 13: Cross-section of polyurethane fiber with glass core (a) and with hollow core (b), respectively, [50].

with inverse opal structure are assembled on ITO conductive glass used in solar cells [52]. It is worth mentioning the fact that an inorganic matrix formed by semiconductors (like $\mathrm{Ge}$, $\mathrm{TiO}_{2}$, InP, and CdSe) or other metal compounds [53] found application in realization of infrared active optical materials due to their high refractive index $[54,55]$, while polymer inverse opals can be used in medicine as scaffolds for tissue engineering or cell culture [56] (Figure 14).

3.2. Sensors. The refractive index is a result of the interplanar distance and the type of packing of the polymer/inorganic spheres according to Bragg's law. Practically, the stop band is directly proportional with the interplanar distance, $d$. In other words, any variation of $d$ should determine a shift in the diffraction peak of the reflected light. This property of the crystalline $3 \mathrm{D}$ arrays makes these materials suitable for optical sensors [1].

Polymer photonic crystals are used in optical sensors, coupled to a transduction device that identifies the optical diffraction shifts that occur once the external medium changes.

Optical sensors based on colloidal crystals (sometimes embedded in hydrogels) are known for their simplicity and low cost and efficiency. In the last years, remarkable results to the preparation of photonic crystal sensors that are highly sensitive to a series of $\mathrm{pH}$ [57], temperature [58], solvent swelling [59], ionic strength, ions [60], and biomolecules [61] have been obtained.

In addition, colloidal crystals were also used to develop new sensors for the detection of endocrine-disrupting chemicals like bisphenol A (BPA) at low costs. Guo et al. [62] explain the concept of photonic crystals-based sensors using combined photonic crystal technology and molecular imprinting techniques. First, poly(methyl methacrylate) spheres were modified with nanocavities derived from the BPA-imprinting method. The modification of the colloidal spheres by these techniques affords nanocavities distributed in the polymeric crystalline array which allow the photonic crystal sensor to identify BPA specifically. Using these procedures the changes in diffraction intensity, which are related to BPA concentrations, can be observed (Figure 15), even at very low concentrations of BPA ranging from $1 \mathrm{ng} / \mathrm{mL}$ to $1 \mu \mathrm{g} / \mathrm{mL}$.
In a recent study, Hu et al. [63] developed an optical sensor sensitive for anions, starting from the properties of an ionic liquid based monomer and photonic crystals.

Therefore, a photopolymerization was conducted using an imidazolium-based ionic liquid monomer (1-(2-acryloyloxyhexyl)-3-methylimidazolium bromide) with methyl methacrylate, a cross-linker and initiator in a solvent mixture (methanol/chloroform), and infiltrated in a photonic crystal consisting of silica colloidal particles. After removal of the inorganic silica particles, a porous inverse opal film was obtained (Figure 16(a)).

The new photonic ionic liquid films were immersed in anionic aqueous solutions. The anions bonded onto the film surface, reducing its hydrophilicity. As a consequence, the volume of the film changed (shrunk) leading to strong modifications of the resulting photonic structures stop bands (Figure 16(b)).

In Figure 16(c), the color changes can also be observed with naked eye, representing a much easier and in-expensive method for identifying anions.

Moreover, it was also possible to identify the polarity of different solvents using these photonic ionic liquid films, due to the interactions between the terminal groups of the ionic liquid and solvent [63].

Although the use of ionic liquids in optical sensors is limited due to time-consuming or difficult synthetic procedures, this new approach based on photonic colloidal crystals afforded a system for rapid, naked-eye detection of anions.

Considering that there are about $10^{8}$ ionic liquids [63] this procedure might allow the development of more ionic liquid monomers for advanced applications in photonic crystals.

3.3. Optical Devices. Recently, the propagation mechanism of light inside 3D polymer colloidal crystals gained much interest, leading to an important progress in photonic applications such as microlasers, waveguides, or photonic crystal fibers [45].

In other words, Furumi et al. [18] fabricated a colloidal crystal film using $200 \mathrm{~nm}$ polystyrene colloidal particles, followed by the thermal polymerization of dimethylsiloxane in the air voids of the 3D structure. Next step consisted in 


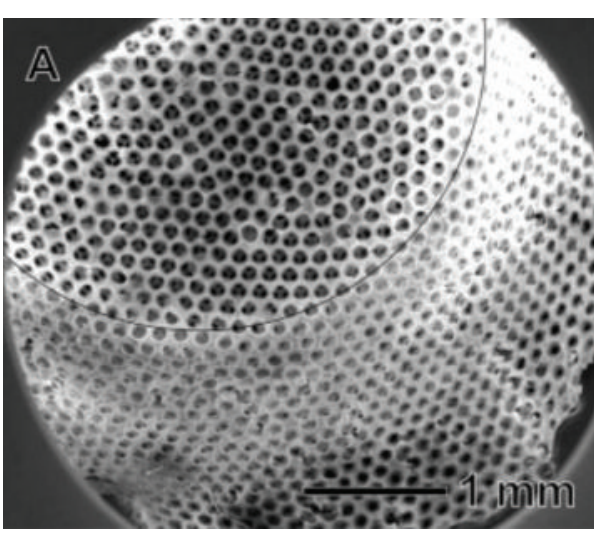

(a)

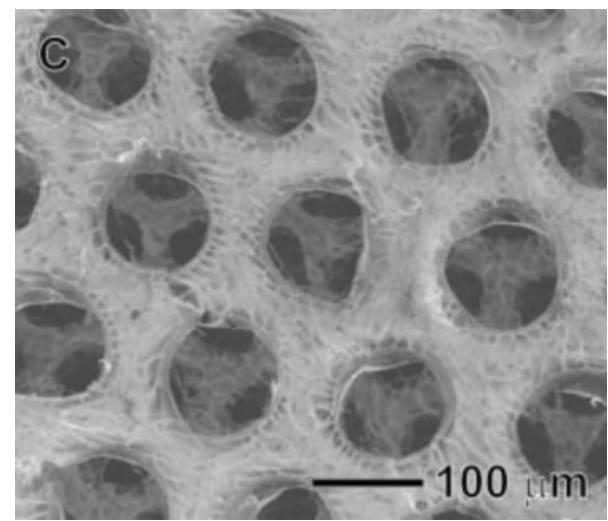

(c)

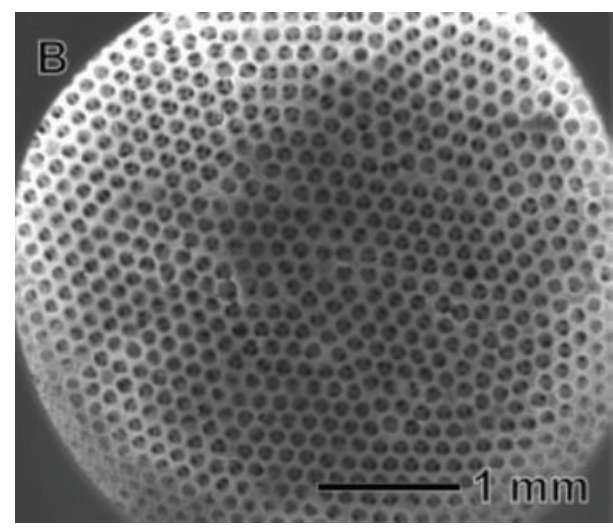

(b)

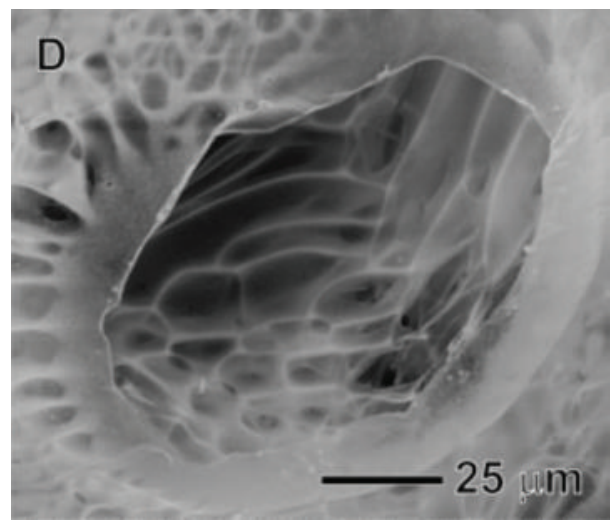

(d)

FIGURE 14: SEM images of the chitosan inverse opal scaffold: (a) side view, (b) top view, (c) a magnified view of the top surface, and (d) a magnified view of the side wall in a pore. The black line in (a) represents the edge of the scaffold [56].

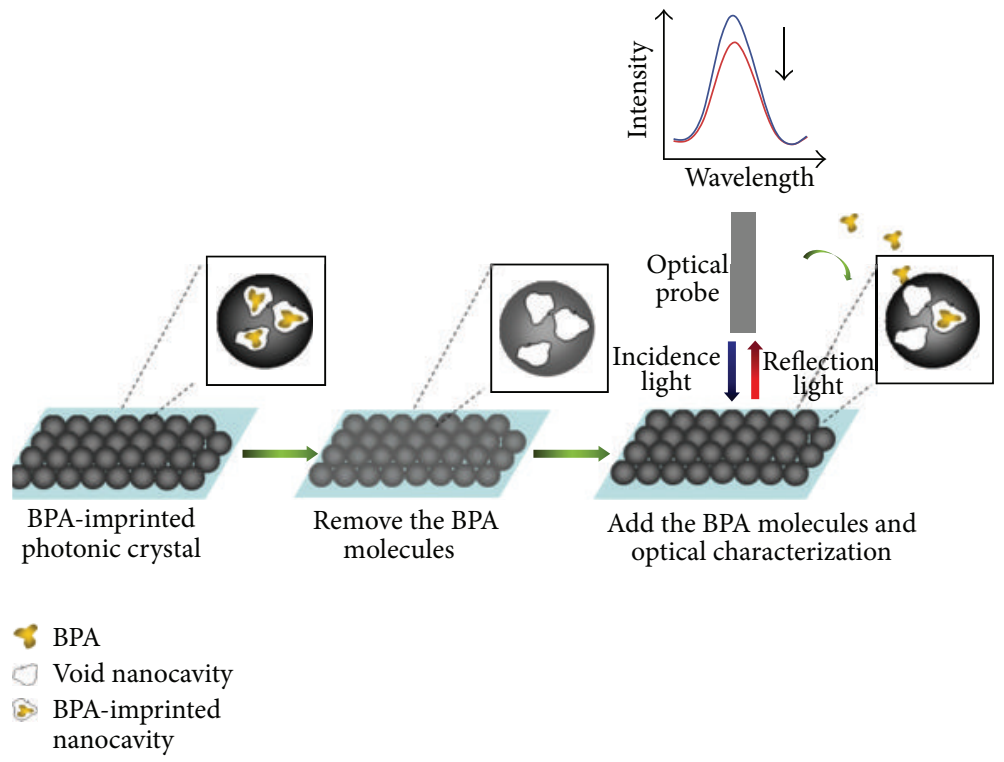

FIGURE 15: Experimental procedure for the detection of BPA using a photonic crystal sensor [62]. 


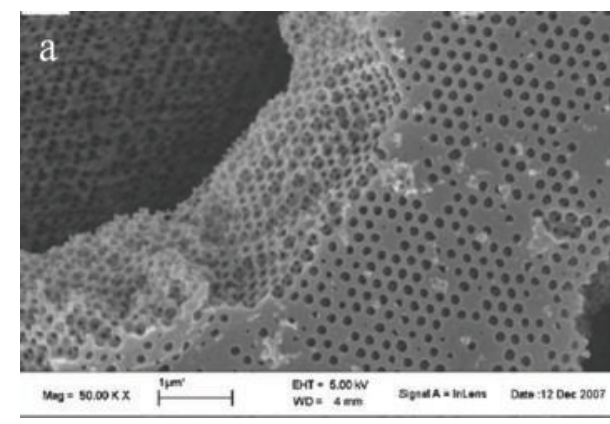

(a)

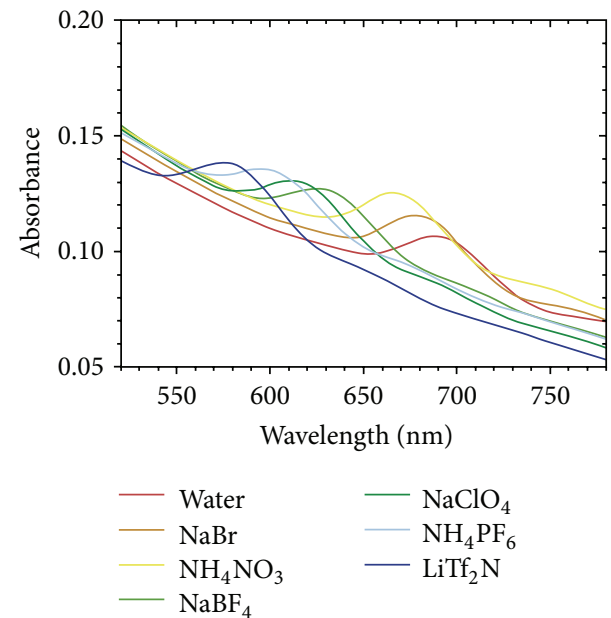

(b)

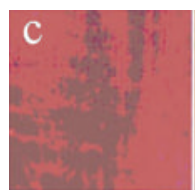

Water

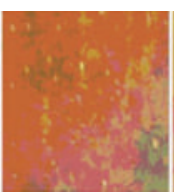

$\mathrm{Br}$

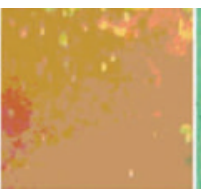

$\mathrm{NO}_{3}{ }^{-}$

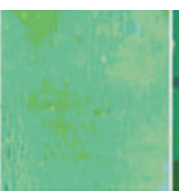

$\mathrm{BF}_{4}^{-}$

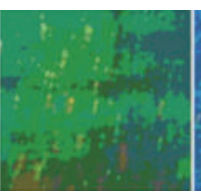

$\mathrm{ClO}_{4}$

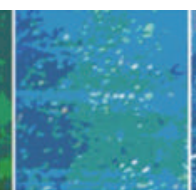

$\mathrm{PF}_{6}$

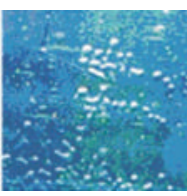

$\mathrm{Tf}_{2} \mathrm{~N}^{-}$

(c)

FIGURE 16: SEM image of the inverseopal photonic ionic liquid film (a). Stop-band shifts of the film upon soaking in various aqueous anionic solutions (b). Color changes of the film after anions aqueous solutions treatment (c) [63].

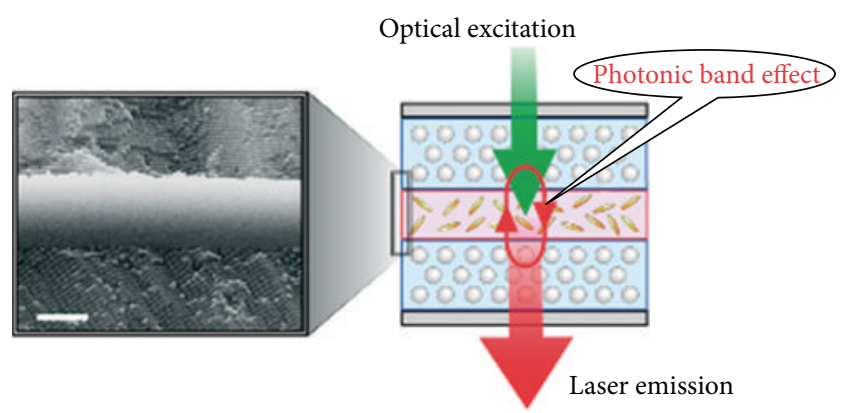

PS particle Fluorescent Rh dye

$\square$ PDMS elastomer $\square$ UV-curable PEG-DA

Figure 17: A schematic illustration of the colloidal crystal laser (CCL) proposed by Furumi et al. A light-emitting planar defect of Rhodamine (Rh)/PEG-DA is sandwiched between a pair of CC films of PS particles stabilized in PDMS elastomer. The left-hand picture represents a cross-sectional scanning electron microscopy (SEM) image of the magnified light-emitting planar defect between the PS/PDMS CC films. The white scale bar represents $2 \mu \mathrm{m}$ [18].

introducing a light emitting planar defect based on fluorescent dye (rhodamine 640) dispersed in a poly(ethylene glycol) diacrylate film in order to obtain a laser effect, resulting in sandwich-type structure (Figure 17).

Therefore, the plastic film through excitation with a green wavelength affords a red laser emission as suggested in Figure 18.

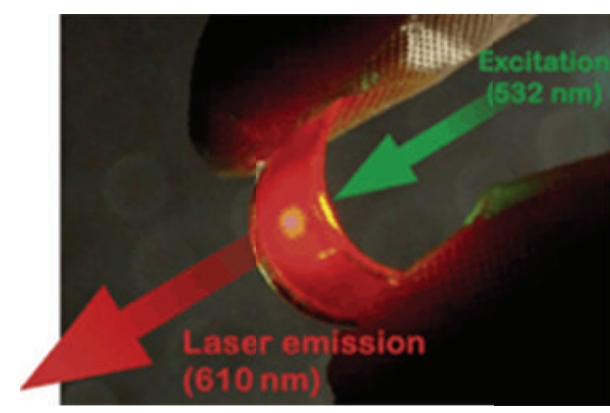

FIgURE 18: Laser effect of the plastic film [18].

Photonic crystal microfibers were obtained by Míguez et al. [64] using an inverted silicon colloidal crystal. First, in rectangular microchannels, colloidal particles of silica ranging from $250 \mathrm{~nm}$ to $900 \mathrm{~nm}$ were self-assembled in FCC structure. After a coating process of the silica spheres in order to control the connectivity between the particles, the microchannels are infiltrated with silicon by a chemical vapor deposition method. The silicon fills the interstitial spaces from the silica colloidal structure. In order to obtain the 3D silicon inverse structure the silica particles are removed, and the resulted microfibers are investigated by SEM and optical microscopy (Figure 19).

In Figure 19(a), the presented microfibers have several micrometers in length. An interesting optical behavior of the microfibers, which due to the various degrees of silicon infiltration exhibit different modulations of the dielectric 

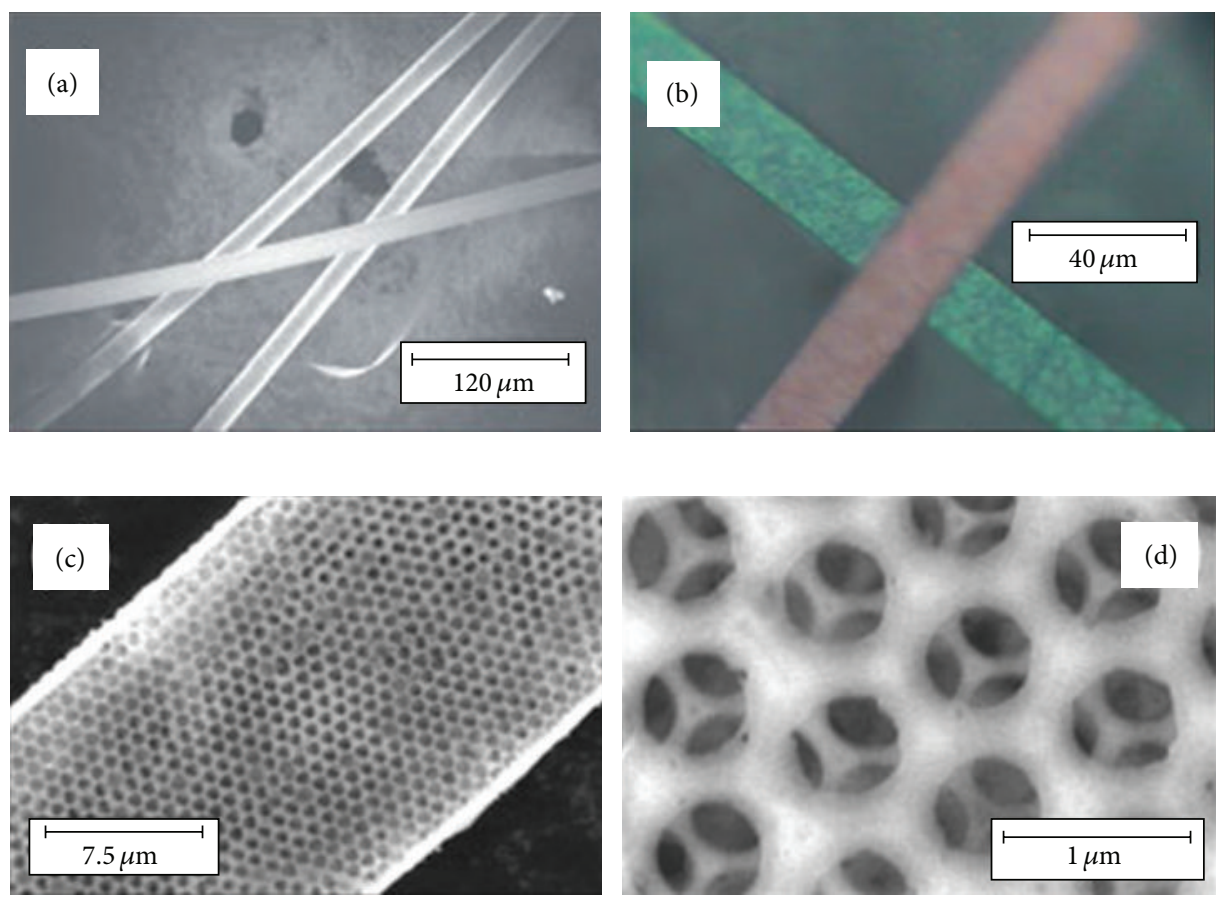

FIGURE 19: Images of inverse silicon microfibers. (a) SEM image of a bunch of fibers collected using a sticky carbon tape; (b) optical picture of two fibers presenting a different degree of infiltration, thus presenting different colors; ((c), (d)) details of the bottom surface of a free standing inverted colloidal crystal fiber showing the high degree of connectivity between the spherical cavities [64].

constant in the structure, thus resulting in distinct reflected colors, is illustrated in Figure 19(b) [64].

Compared to inorganic fibers, this method provided a simple synthesis procedure for obtaining microfibers with higher flexibility and controllable optical properties. As the authors suggested [64], the optical properties of the synthesized photonic crystal microfibers could make them suitable in future for optical components in microcomputers replacing the conventional semiconductor nanowires.

In conclusion, the aim of this review has been to briefly present the concept of photonic crystals and the advantages of obtaining these structures using polymeric materials. The property of self-assembling remains one of the key characteristics that motivate the use of these materials. Control of the self-assembly process as well as of the morphology attained allows the elaboration of new materials capable of interacting with light and able to be incorporated into novel devices.

The doping with various dyes, the interaction with inorganic particles in hybrid systems innovative synthesis techniques for specific applications (molecularly imprinted polymers, synthesis in the presence of ionic liquids) constitute new topics for novel and innovative applications based on polymeric colloidal particles and photonic crystals.

\section{Acknowledgment}

The authors would like to acknowledge thank for the financial support provided by the National Authority for Scientific Research from the Ministry of Education, Research and
Youth of Romania through the PN-II-PT-PCCA-2011-3.20042-RPETUM Project.

\section{References}

[1] Y. Xia, B. Gates, Y. Yin, and Y. Lu, "Monodispersed colloidal spheres: old materials with new applications," Advanced Materials, vol. 12, no. 10, pp. 693-713, 2000.

[2] Y. Chen, W. T. Ford, N. F. Materer, and D. Teeters, "Conversion of colloidal crystals to polymer nets: turning latex particles inside out," Chemistry of Materials, vol. 13, no. 8, pp. 2697-2704, 2001.

[3] J. Zhang, Z. Sun, and B. Yang, "Self-assembly of photonic crystals from polymer colloids," Current Opinion in Colloid and Interface Science, vol. 14, no. 2, pp. 103-114, 2009.

[4] S. A. Rinne, F. García-Santamaría, and P. V. Braun, "Embedded cavities and waveguides in three-dimensional silicon photonic crystals," Nature Photonics, vol. 2, no. 1, pp. 52-56, 2008.

[5] A. Herzog Cardoso, C. A. P. Leite, M. E. D. Zaniquelli, and F. Galembeck, "Easy polymer latex self-assembly and colloidal crystal formation: the case of poly[styrene-co-(2-hydroxyethyl methacrylate)]," Colloids and Surfaces A, vol. 144, no. 1-3, pp. 207-217, 1998.

[6] L. Rayleigh, "XXVI. On the remarkable phenomenon of crystalline reflexion described by Prof. Stokes," Philosophical Magazine Series 5, vol. 26, no. 160, pp. 256-265, 1888.

[7] E. Yablonovitch, "Inhibited spontaneous emission in solid-state physics and electronics," Physical Review Letters, vol. 58, no. 20, pp. 2059-2062, 1987.

[8] S. John, "Strong localization of photons in certain disordered dielectric superlattices," Physical Review Letters, vol. 58, no. 23, pp. 2486-2489, 1987. 
[9] G. I. N. Waterhouse and M. R. Waterland, "Opal and inverse opal photonic crystals: fabrication and characterization," Polyhedron, vol. 26, no. 2, pp. 356-368, 2007.

[10] X. Zhao, Y. Cao, F. Ito et al., "Colloidal crystal beads as supports for biomolecular screening," Angewandte Chemie, vol. 45, no. 41, pp. 6835-6838, 2006.

[11] E. Rusen, A. Mocanu, A. Diacon, and B. Marculescu, "Fluorescence enhancement of rhodamine $B$ in the presence of photonic crystal heterostructures," Journal of Physical Chemistry C, vol. 115, no. 30, pp. 14947-14953, 2011.

[12] Y. Zhao, X. Zhao, and Z. Gu, "Photonic crystals in bioassays," Advanced Functional Materials, vol. 20, no. 18, pp. 2970-2988, 2010.

[13] A. Mocanu, B. Marculescu, R. Somoghi, F. Miculescu, C. Boscornea, and I. C. Stancu, "Fluorescence enhancement for the complex PAMAM-BSA in the presence of photonic crystal heterostructures," Colloids and Surfaces A, vol. 392, no. 1, pp. 288-293, 2011.

[14] A. C. Arsenault, D. P. Puzzo, A. Ghoussoub, I. Manners, and G. A. Ozin, "Development of photonic crystal composites for display applications," Journal of the Society for Information Display, vol. 15, no. 12, pp. 1095-1098, 2007.

[15] A. C. Arsenault, D. P. Puzzo, I. Manners, and G. A. Ozin, "Photonic-crystal full-colour displays," Nature Photonics, vol. 1, no. 8, pp. 468-472, 2007.

[16] Z. Ye, J. M. Park, K. Constant, T. G. Kim, and K. M. Ho, "Photonic crystal: energy-related applications," Journal of Photonics for Energy, vol. 2, no. 1, Article ID 021012, 2012.

[17] J. R. Lawrence, Y. Ying, P. Jiang, and S. H. Foulger, "Dynamic tuning of organic lasers with colloidal crystals," Advanced Materials, vol. 18, no. 3, pp. 300-303, 2006.

[18] S. Furumi, H. Fudouzi, H. T. Miyazaki, and Y. Sakka, "Flexible polymer colloidal-crystal lasers with a light-emitting planar defect," Advanced Materials, vol. 19, no. 16, pp. 2067-2072, 2007.

[19] J. Ge and Y. Yin, "Responsive photonic crystals," Angewandte Chemie, vol. 50, no. 7, pp. 1492-1522, 2011.

[20] C. I. Aguirre, E. Reguera, and A. Stein, "Tunable colors in opals and inverse opal photonic crystals," Advanced Functional Materials, vol. 20, no. 16, pp. 2565-2578, 2010.

[21] S. Furumi, H. Fudouzi, and T. Sawada, "Self-organized colloidal crystals for photonics and laser applications," Laser and Photonics Reviews, vol. 4, no. 2, pp. 205-220, 2010.

[22] C. T. Chan and J. A. Yeh, "Tunable photonic crystal based on capillary attraction and repulsion," Optics Express, vol. 18, no. 20, pp. 20894-20899, 2010.

[23] S. S. Mishra and V. K. Singh, "Comparative study of fundamental properties of honey comb photonic crystal fiber at $1.55 \mu \mathrm{m}$ wavelength," Journal of Microwaves, Optoelectronics and Electromagnetic Applications, vol. 10, no. 2, pp. 343-354, 2011.

[24] Y. Chen and S. Sajjadi, "Particle formation and growth in ab initio emulsifier-free emulsion polymerisation under monomerstarved conditions," Polymer, vol. 50, no. 2, pp. 357-365, 2009.

[25] D. Suzuki, J. G. McGrath, H. Kawaguchi, and L. A. Lyon, "Colloidal crystals of thermosensitive, core/shell hybrid microgels," Journal of Physical Chemistry C, vol. 111, no. 15, pp. 5667-5672, 2007.

[26] X. Xu and S. A. Asher, "Synthesis and utilization of monodisperse hollow polymeric particles in photonic crystals," Journal of the American Chemical Society, vol. 126, no. 25, pp. 79407945, 2004.
[27] S. M. Klein, V. N. Manoharan, D. J. Pine, and F. F. Lange, "Preparation of monodisperse PMMA microspheres in nonpolar solvents by dispersion polymerization with a macromonomeric stabilizer," Colloid and Polymer Science, vol. 282, no. 1, pp. 7-13, 2003.

[28] J. W. Kim, J. H. Ryu, and K. D. Suh, "Monodisperse micronsized macroporous poly(styrene-co-divinylbenzene) particles by seeded polymerization," Colloid and Polymer Science, vol. 279, no. 2, pp. 146-152, 2001.

[29] X. Wu, A. Yamilov, X. Liu et al., "Ultraviolet photonic crystal laser," Applied Physics Letters, vol. 85, no. 17, pp. 3657-3659, 2004.

[30] E. A. Wood, Crystals and Light: An Introduction to Optical Crystallography, Dover Publications, 1977.

[31] E. Rusen, A. Mocanu, C. Corobea, and B. Marculescu, "Obtaining of monodisperse particles through soap-free polymerization in the presence of C60," Colloids and Surfaces A, vol. 355, no. 1-3, pp. 23-28, 2010.

[32] H. Fudouzi, "Novel coating method for artificial opal films and its process analysis," Colloids and Surfaces A, vol. 311, no. 1-3, pp. 11-15, 2007.

[33] H. Fudouzi, "Fabricating high-quality opal films with uniform structure over a large area," Journal of Colloid and Interface Science, vol. 275, no. 1, pp. 277-283, 2004.

[34] X. He, Y. Thomann, R. J. Leyrer, and J. Rieger, "Iridescent colors from films made of polymeric core-shell particles," Polymer Bulletin, vol. 57, no. 5, pp. 785-796, 2006.

[35] M. Allard, E. H. Sargent, E. Kumacheva, and O. Kalinina, "Characterization of internal order of colloidal crystals by optical diffraction," Optical and Quantum Electronics, vol. 34, no. 1-3, pp. 27-36, 2002.

[36] P. Jiang, G. N. Ostojic, R. Narat, D. M. Mittleman, and V. L. Colvin, "The fabrication and bandgap engineering of photonic multilayers," Advanced Materials, vol. 13, no. 6, pp. 389-393, 2001.

[37] H. Fudouzi, "Optical properties caused by periodical array structure with colloidal particles and their applications," Advanced Powder Technology, vol. 20, no. 5, pp. 502-508, 2009.

[38] S. Reculusa, P. Massé, and S. Ravaine, "Three-dimensional colloidal crystals with a well-defined architecture," Journal of Colloid and Interface Science, vol. 279, no. 2, pp. 471-478, 2004.

[39] H. Zhou, S. Xu, Z. Sun, X. Du, and J. Xie, "Rapid determination of colloidal crystal's structure by reflection spectrum," Colloids and Surfaces A, vol. 375, no. 1-3, pp. 50-54, 2011.

[40] A. Emoto, E. Uchida, and T. Fukuda, "Fabrication and optical properties of binary colloidal crystal monolayers consisting of micro- and nano-polystyrene spheres," Colloids and Surfaces A, vol. 396, pp. 189-194, 2012.

[41] Z. Z. Gu, H. Chen, S. Zhang, L. Sun, Z. Xie, and Y. Ge, "Rapid synthesis of monodisperse polymer spheres for self-assembled photonic crystals," Colloids and Surfaces A, vol. 302, no. 1-3, pp. 312-319, 2007.

[42] E. Yablonovitch, T. J. Gmitter, and K. M. Leung, "Photonic band structure: the face-centered-cubic case employing nonspherical atoms," Physical Review Letters, vol. 67, no. 17, pp. 2295-2298, 1991.

[43] W. H. Wong, K. K. Liu, K. S. Chan, and E. Y. B. Pun, "Polymer devices for photonic applications," Journal of Crystal Growth, vol. 288, no. 1, pp. 100-104, 2006.

[44] P. S. J. Russell, "Photonic-crystal fibers," Journal of Lightwave Technology, vol. 24, no. 12, pp. 4729-4749, 2006. 
[45] K. Hansen and H. Imam, "Photonic crystal fiber," Optik \& Photonik, vol. 5, no. 2, pp. 37-41, 2010.

[46] Z. Z. Gu, H. Xu, P. Wu, C. Zhu, and A. Elbaz, "Photonic crystal for gas sensing," Journal of Materials Chemistry C, 2013.

[47] Z. Cai, Y. J. Liu, J. Teng, and X. Lu, "Fabrication of large domain crack-free colloidal crystal heterostructures with superposition bandgaps using hydrophobic polystyrene spheres," ACS Applied Materials \& Interfaces, vol. 4, no. 10, pp. 5562-5569, 2012.

[48] H. Huang, J. Chen, Y. Yu, Z. Shi, H. Möhwald, and G. Zhang, "Controlled gradient colloidal photonic crystals and their optical properties," Colloids and Surfaces A, vol. 428, pp. 9-17, 2013.

[49] Y. Li, Z. Sun, J. Zhang et al., "Polystyrene@ $\mathrm{TiO}_{2}$ core-shell microsphere colloidal crystals and nonspherical macro-porous materials," Journal of Colloid and Interface Science, vol. 325, no. 2, pp. 567-572, 2008.

[50] J. H. Moon, G. R. Yi, and S. M. Yang, "Fabrication of hollow colloidal crystal cylinders and their inverted polymeric replicas," Journal of Colloid and Interface Science, vol. 287, no. 1, pp. 173-177, 2005.

[51] O. Kalinina and E. Kumacheva, "Polymeric nanocomposite material with a periodic structure," Chemistry of Materials, vol. 13, no. 1, pp. 35-38, 2001.

[52] G. Cao and C. J. Brinker, Annual Review of Nano Research, World Scientific, 2006.

[53] M. Lanata, M. Cherchi, A. Zappettini, S. M. Pietralunga, and M. Martinelli, "Titania inverse opals for infrared optical applications," Optical Materials, vol. 17, no. 1-2, pp. 11-14, 2001.

[54] C. Lü, C. Guan, Y. Liu, Y. Cheng, and B. Yang, "PbS/polymer nanocomposite optical materials with high refractive index," Chemistry of Materials, vol. 17, no. 9, pp. 2448-2454, 2005.

[55] T. Huang, Q. Zhao, J. Xiao, and L. Qi, "Controllable selfassembly of pbs nanostars into ordered structures: close-packed arrays and patterned arrays," ACS Nano, vol. 4, no. 8, pp. 47074716, 2010.

[56] S. W. Choi, J. Xie, and Y. Xia, "Chitosan-based inverse opals: three-dimensional scaffolds with uniform pore structures for cell culture," Advanced Materials, vol. 21, no. 29, pp. 2997-3001, 2009.

[57] K. Lee and S. A. Asher, "Photonic crystal chemical sensors: $\mathrm{pH}$ and ionic strength," Journal of the American Chemical Society, vol. 122, no. 39, pp. 9534-9537, 2000.

[58] J. Ballato and A. James, "A ceramic photonic crystal temperature sensor," Journal of the American Ceramic Society, vol. 82, no. 8, pp. 2273-2275, 1999.

[59] H. Fudouzi and Y. Xia, "Colloidal crystals with tunable colors and their use as photonic papers," Langmuir, vol. 19, no. 23, pp. 9653-9660, 2003.

[60] H. C. Kolb, M. G. Finn, and K. B. Sharpless, "Click chemistry: diverse chemical function from a few good reactions," Angewandte Chemie, vol. 40, no. 11, pp. 2004-2021, 2001.

[61] S. Mandal, J. M. Goddard, and D. Erickson, "A multiplexed optofluidic biomolecular sensor for low mass detection," Lab on a Chip, vol. 9, no. 20, pp. 2924-2932, 2009.

[62] C. Guo, C. Zhou, N. Sai et al., "Detection of bisphenol A using an opal photonic crystal sensor," Sensors and Actuators B, vol. 166-167, pp. 17-23, 2012.

[63] X. Hu, J. Huang, W. Zhang, M. Li, C. Tao, and G. Li, "Photonic ionic liquids polymer for naked-eye detection of anions," Advanced Materials, vol. 20, no. 21, pp. 4074-4078, 2008.
[64] H. Míguez, S. M. Yang, N. Tétreault, and G. A. Ozin, “Oriented free-standing three-dimensional silicon inverted colloidal photonic crystal microfibers," Advanced Materials, vol. 14, no. 24, pp. 1805-1808, 2002.

[65] E. Rusen, A. Mocanu, and B. Marculescu, "Obtaining of monodisperse particles through soap-free and seeded polymerization, respectively, through polymerization in the presence of C 60," Colloid and Polymer Science, vol. 288, no. 7, pp. 769-776, 2010.

[66] D. Gaillot, T. Yamashita, and C. J. Summers, "Photonic band gaps in highly conformal inverse-opal based photonic crystals," Physical Review B, vol. 72, no. 20, Article ID 205109, 2005. 

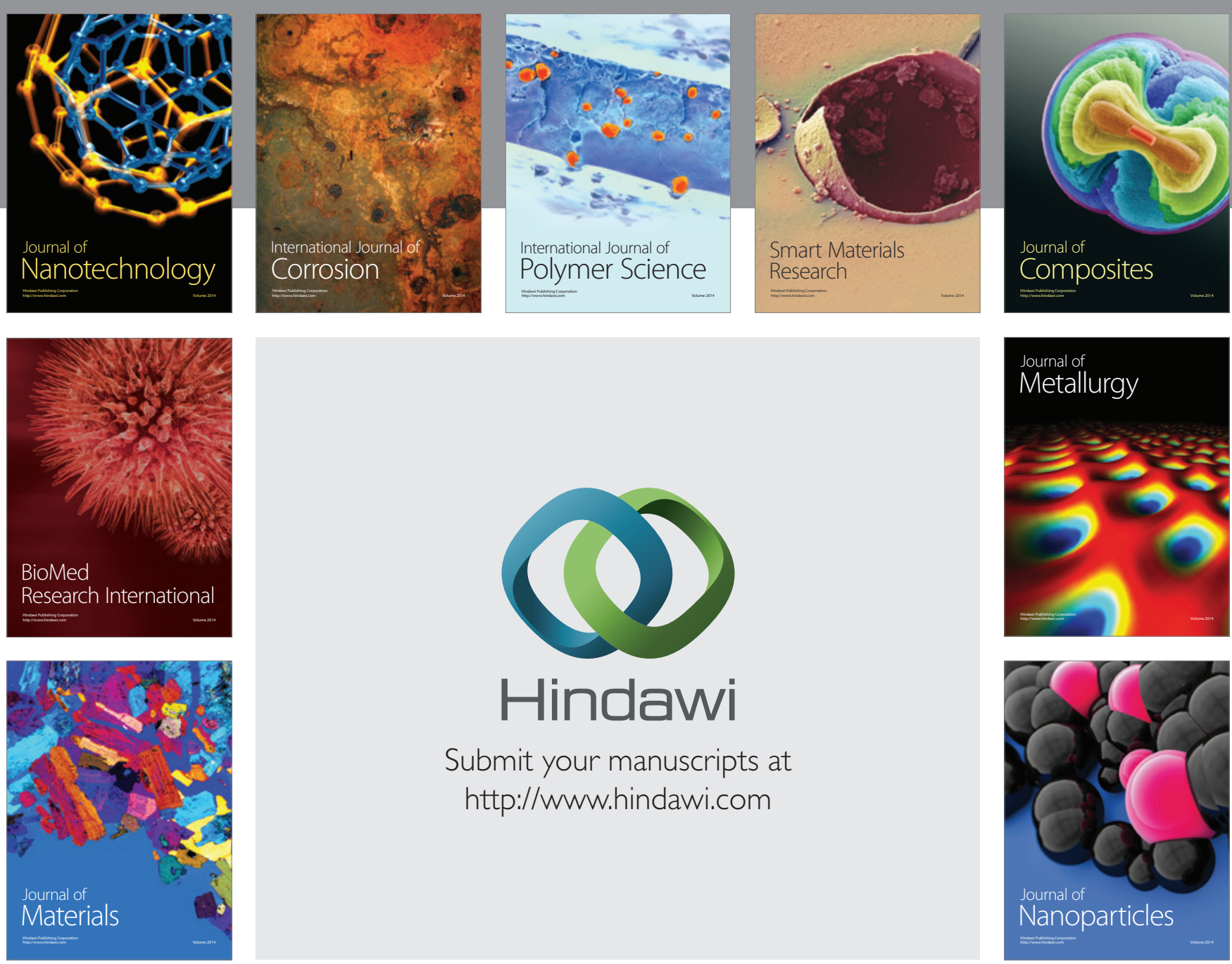

Submit your manuscripts at http://www.hindawi.com
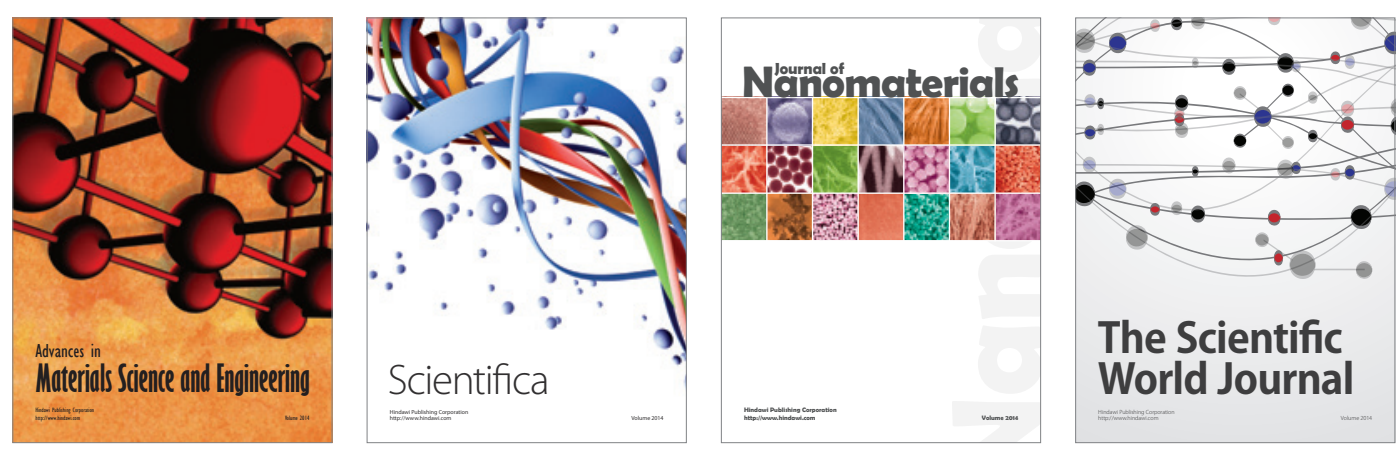

\section{The Scientific World Journal}
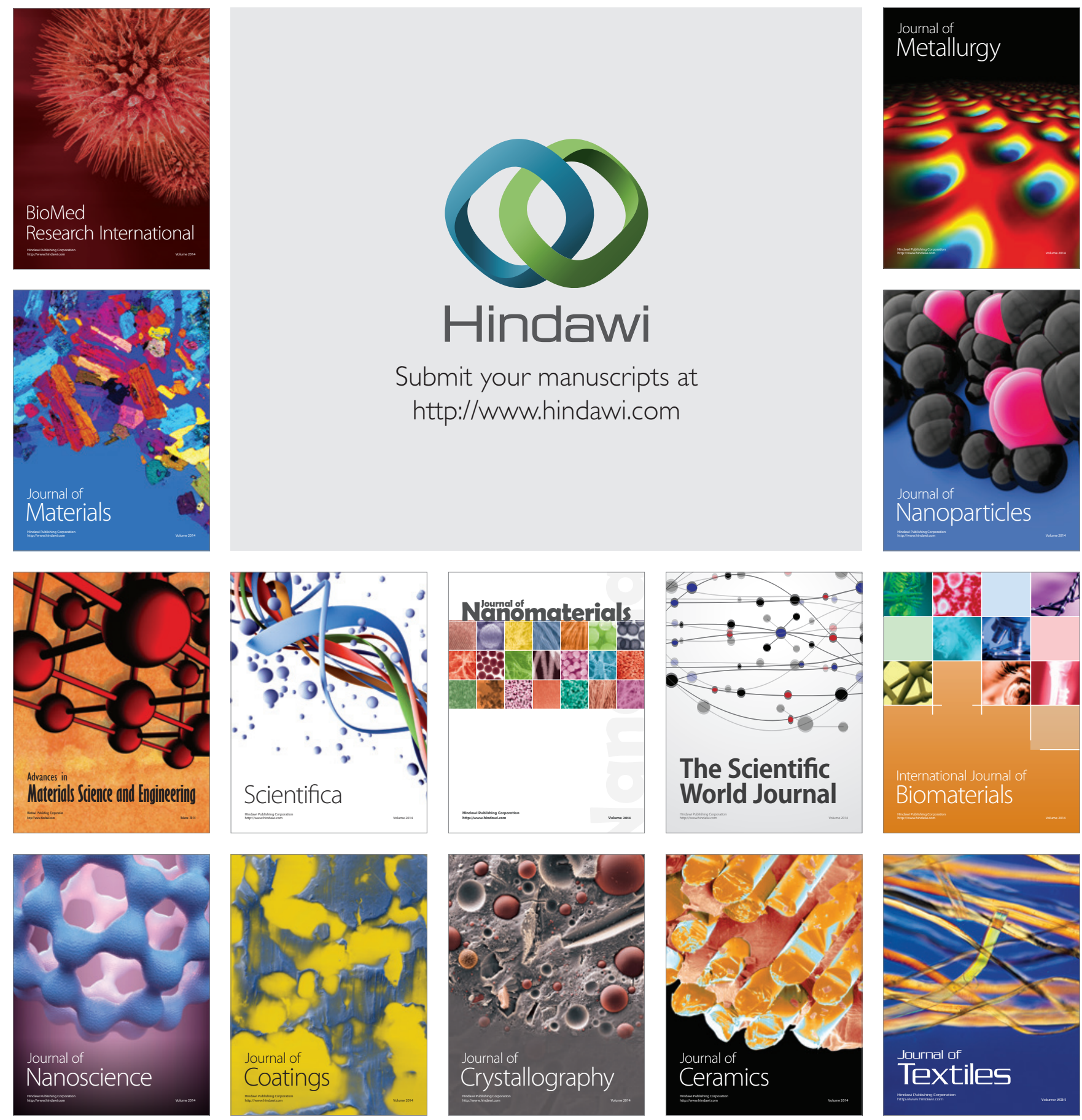